\title{
Dépôts et phénomènes éoliens sur l'île de Banks, Territoires du Nord-Ouest, Canada
}

\author{
A. PISSART \\ Laboratoire de géologie et de géographie physique, Université de Liège, \\ 7 , place du 20 Aồt, Liège, Belgique \\ ET \\ J.-S. VInCENT ET S. A. EDLUND \\ Commission géologique du Canada, 401, rue LeBreton, Ottawa, Ont., Canada K1A OE8 \\ Reçu le 6 mai 1977 \\ Revision acceptée pour publication le 18 mai 1977
}

\begin{abstract}
En plusieurs endroits de l'île de Banks, des actions non négligeables de déflation se produisent actuellement. La déflation s'exerce sur les alluvions des plaines alluviales et des plaines d'épandages fluvio-glaciaires, sur des sables éoliens précédemment stabilisés et aussi quelquefois directement sur des accumulations sableuses d'âge mésozoïque et tertiaire. Localement, les vents directement sur des accumulations sableuses d'âge mésozoïque et tertiare. Localement, les vents ont façonné des cailloux éolisés typiques.

La couverture végétale des surfaces soumises aux actions éoliennes est décrite en précisant les différentes espèces de plantes qui ont été observées.

Des dépôts éoliens du cours inférieur de la rivière Thomsen sont étudiés en détail. L'étude d'une coupe a montré que des polygones de fente de gel se sont développés pendant l'accumulation des sables. Elle établit, en outre, que des lentilles de glace de ségrégation peuvent se former à la partie supérieure du pergélisol, lorsque celui-ci s'élève suite à l'accumulation en surface de dépôts éoliens.

Deux datations au radiocarbone ont révélé que la sédimentation éolienne dans deux sites différents le long de la rivière Thomsen a débuté il y a $3790 \pm 90$ ans BP (GSC-2119) et $3460 \pm 80$ ans BP (GSC-2124). Des âges de $5800 \pm 180$ ans BP (GSC-2242) et de $8430 \pm 120$ ans BP (GSC-2419) ont été respectivement obtenus pour le début de la sédimentation éolienne le long de la rivière Bernard et sur la plaine d'épandage au sud-est de Sachs Harbour. En guise d'hypothèse il est avancé que l'initiation de l'activité éolienne, il y a environ 4000 ans, pourrait avoir été engendrée par un refroidissement du climat et un régime météorologique plus sec sur l'île de Banks.
\end{abstract}

At the present time, eolian processes are active in many localities on Banks Island. Sediments of alluvial and outwash plains, as well as previously stabilized areas of eolian sands and areas where sandy Mesozoic and Tertiary deposits crop out, are subject to deflation. Locally ventifacts have been faceted.

The vegetation cover of surfaces subject to eolian activity is described, as well as the main species observed on these surfaces.

The eolian deposits of lower Thomsen River are described in detail. The study of a section has shown that ice-wedge polygons developed during periods of sand accumulation. The formation of segregation ice at the top of the permafrost, when it is raised because of the accumulation of eolian sands on the surface, is outlined.

Two radiocarbon age determinations from two different sites along Thomsen River show that the deposition of eolian sands began $3790 \pm 90$ years BP (GSC-2119) and $3460 \pm 80$ years BP (GSC 2124). Ages of $5800 \pm 180$ years BP (GSC-2242) and $8430 \pm 120$ years BP (GSC-2419) were obtained respectively for the start of eolian sedimentation along Bernard River and on the outwash surface situated southeast of Sachs Harbour. The hypothesis that the initiation of eolian activity around 4000 years ago may have been the result of a drier and cooler climate on Banks Island is put forward.

Can. J. Earth Sci, , 14, 2462-2480 (1977)

\section{Introduction}

Peu de publications ont été consacrées à l'action du vent dans les régions à pergélisol continu, à faibles précipitations et à végétation de toundra de l'Arctique canadien. Bird (1967, pp. 237-241) s'est efforcé de donner une vue d'ensemble des connaissances sur le sujet. Selon lui, les actions éoliennes actuelles sont en général locales et peu importantes. Il précise cependant diverses régions où des dunes de sable existent 
et où les phénomènes éoliens ne sont pas négligeables. La zone où les formes éoliennes sont les plus apparentes est située au nord de la rivière Thelon; le matériel remanié par le vent provient du substratum sableux et aussi des dépôts glaciaires locaux qui sont très sableux. A l'ouest de la baie de Pelly, les deltas des rivières sont aussi la source de sédiments éoliens. Des nuages de poussières sont observés parfois dans le district du Mackenzie à partir de silt marin et de till silteux.

Sur les îles réparties au nord du Canada, les articles qui traitent des phénomènes éoliens sont moins nombreux encore. Des cailloux éolisés ont été signalés au nord-ouest de l'île de Baffin (Bird 1967), et au sud de l'île Bylot (Drury 1962). Des nappes sableuses se sont formées sur l'île Victoria à partir de moraines et de plaines d'épandages (Bird 1967). Toutefois, Washburn (1947), St-Onge (1965) et Pissart (1966) ont précisé que les phénomènes de déflation et les dépôts de particules minérales étaient fort peu actifs respectivement sur les îles Victoria, Ellef Ringnes et Prince Patrick. Corbel (1958) a, de la même manière, écrit que près d'Alert à proximité de l'extrémité nord de l'île d'Ellesmere, les actions d'éolisation étaient extrêmement faibles.

St-Onge (1965) et Pissart (1966) ont cependant indiqué que l'action géomorphologique du vent est importante sur ces îles par le rôle qu'il joue en accumulant localement la neige, accumulations qui contrôlent les phénomènes de nivation. French (1972) a détaillé comment les bancs de neige entassés par le vent sont à l'origine des vallées asymétriques du nord-ouest de l'île de Banks. Cet article nous paraît être le seul qui traite d'actions éoliennes se produisant de nos jours sur l'île de Banks.

Dans plusieurs manuels (Tricart et Cailleux 1961; Embleton et King 1968), l'action éolienne est considérée comme très efficace dans les régions périglaciaires. Cette opinion est justifiée par le fait que dans les régions arctiques l'action du vent est favorisée par l'absence locale de couverture végétale, la fréquence des vents violents, la faiblesse des précipitations et l'existence de grandes quantités de sédiments fins produits par les glaciations. Les connaissances publiées actuellement sur le grand nord canadien ne paraissent pas confirmer cette hypotièse. Nous en chercherons l'explication après avoir décrit les principales actions éoliennes sur l'île de Banks, située à l'extrémité sud-ouest de l'archipel arctique canadien (figs 1,2).

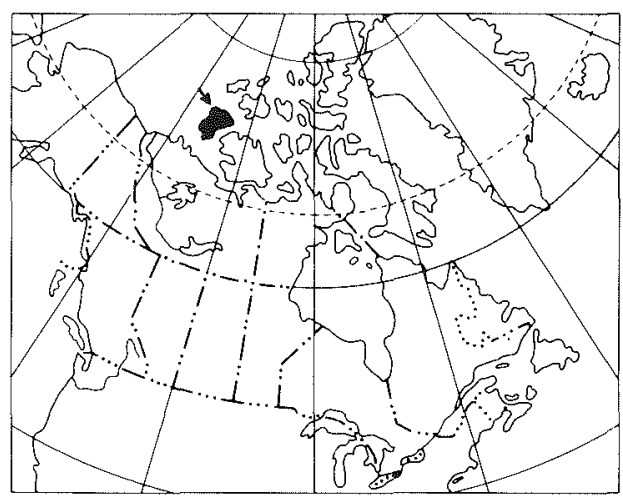

FIG. 1. Localisation de l'île de Banks.

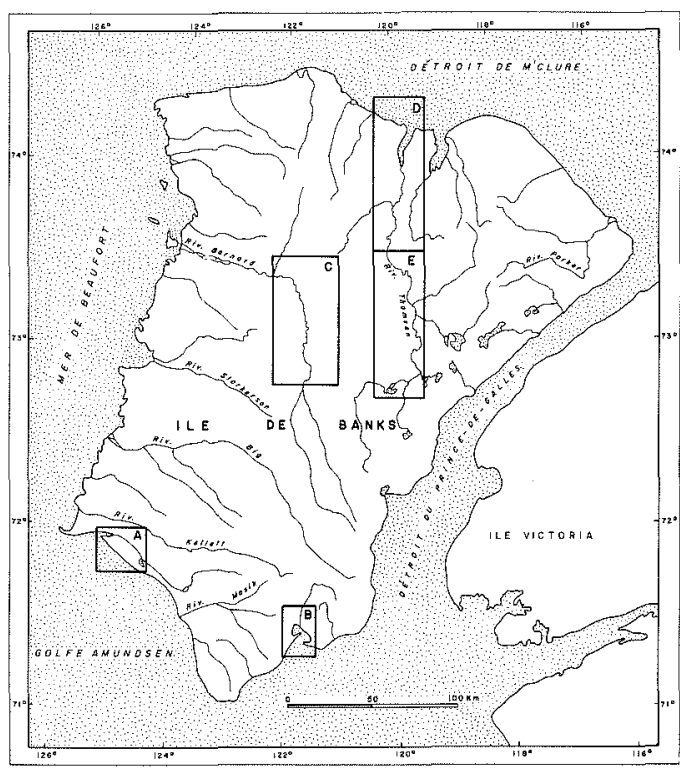

FIG. 2. Carte de l'île de Banks donnant la localisation des cartes détaillées de la fig. 3 .

\section{Données sur les vents de l'île de Banks}

Deux stations météorologiques, la première à Sachs Harbour au sud-oue t de l'île et la seconde à Johnson Point sur la côte est, enregistrent des observations sur les vents. ${ }^{1}$ Le tableau 1 résume les données obtenues à la station de Sachs Harbour de 1970 à 1974.

La station de Johnson Point a recueilli des données entre 1972 et 1975 . Ces données ont les mêmes ordres de grandeur que celles de la station de Sachs Harbour. Aux environs de cette der-

\footnotetext{
${ }^{1}$ Ces données sont publiées mensuellement, par le Ministère de l'environnement du Canada, dans une brochure intitulée: Résumé mensuel-Données météorologiques pour le Canada.
} 
TABLeAu 1. Données sur les vents pour la période 1970-1974 station météorologique de Sachs Harbour

\begin{tabular}{|c|c|c|c|c|c|c|c|}
\hline \multirow[b]{2}{*}{ Mois } & \multicolumn{4}{|c|}{$\begin{array}{l}\text { Fréquence mensuelle des vitesses en nombre } \\
\text { de jours* }\end{array}$} & \multirow{2}{*}{$\begin{array}{c}\text { Vitesse } \\
\text { mensuelle } \\
\text { moyenne } \\
(\mathrm{km} / \mathrm{h})\end{array}$} & \multirow{2}{*}{$\begin{array}{c}\text { Vitesse } \dagger \\
\text { maximale } \\
\text { du mois } \\
(\mathrm{km} / \mathrm{h})\end{array}$} & \multirow{2}{*}{$\begin{array}{c}\text { Vitesset de } \\
\text { la rafale } \\
\text { maximale } \\
\text { du mois } \\
(\mathrm{km} / \mathrm{h})\end{array}$} \\
\hline & $\begin{array}{r}>62 \\
\mathrm{~km} / \mathrm{h}\end{array}$ & $\begin{array}{l}20-61 \\
\mathrm{~km} / \mathrm{h}\end{array}$ & $\begin{array}{r}1-19 \\
\mathrm{~km} / \mathrm{h}\end{array}$ & calmes & & & \\
\hline Janvier & 0.6 & 10.6 & 18.8 & 1.0 & 18.7 & 80 & 112 \\
\hline Février & 0.0 & 7.4 & 19.4 & 1.4 & 16.1 & 61 & 77 \\
\hline Mars & 0.2 & 12.4 & 16.8 & 1.6 & 18.8 & 72 & - \\
\hline Avril & 0.0 & 14.8 & 13.2 & 2.0 & 19.7 & 66 & 79 \\
\hline Mai & 0.0 & 14.4 & 16.2 & 0.4 & 19.7 & 58 & 64 \\
\hline Juin & 0.0 & 12.8 & 16.0 & 1.2 & 18.1 & 51 & 58 \\
\hline Juillet & 0.0 & 15.4 & 15.4 & 0.2 & 19.8 & 61 & 72 \\
\hline Août & 0.0 & 17.4 & 13.0 & 0.6 & 25.5 & 66 & 100 \\
\hline Septembre & 0.0 & 14.8 & 14.6 & 0.6 & 21.1 & 71 & 71 \\
\hline Octobre & 0.2 & 16.4 & 13.6 & 0.8 & 22.3 & 74 & 85 \\
\hline Novembre & 0.2 & 11.8 & 16.2 & 1.8 & 19.3 & 80 & 105 \\
\hline Décembre & 0.0 & 11.6 & 17.2 & 2.2 & 17.5 & 72 & 84 \\
\hline Moyenne & 0.1 & 13.3 & 15.9 & 1.2 & 18.4 & - & - \\
\hline
\end{tabular}

* Observation horaire régulière de $17 \mathrm{~h}$.
+ Mesure maximale pour un mois donné sur une période de 5 ans.

nière station, les vents sont en moyenne plus forts à la fin de l'été et en automne, bien que les vents violents apparaissent surtout en hiver. I1 existe cependant tout au cours de l'année des vents suffisamment puissants pour mobiliser des silts et des sables. Dans le cas des deux stations, la direction des vents dominants correspond très étroitement à la direction des vents responsables des vitesses maximales.

Il est toutefois difficile d'extrapoler, à partir des données des deux stations en bordure de la mer, la direction des vents à l'intérieur des terres. Par exemple, les données météorologiques recueillies au centre de l'île $\left(72^{\circ} 17^{\prime} 20^{\prime \prime} \mathrm{N}\right.$ et $120^{\circ} 42^{\prime} 00^{\prime \prime} \mathrm{W}$ ), au cours de l'été 1974 , indiquent une direction prédominante des vents variant entre l'ouest-sud-ouest et l'ouest, alors que la direction dominante, pour la même période, à Sachs Harbour était sud-sud-est et à Johnson Point est-nord-est.

\section{Répartition des milieux d'actions éoliennes actuelles et circonstances locales}

Les photographies aériennes montrent sous forme de taches blanches les étendues où la déflation contemporaine est assez intense pour que n'existe pratiquement aucune couverture végétale. Une série de cartes (figs $3 \mathrm{~A}-3 \mathrm{E}$ ) basées en premier lieu sur des observations de terrain obtenues au cours de la cartographie systématique des dépôts superficiels de l'île de Banks et en second lieu sur l'interprétation de photographies aériennes au 1:100000 ont été réalisées par le second auteur. Elles montrent la répartition de toutes les régions où l'action éolienne est présentement intense, et ceci pour la totalité de l'île. Généralement il est possible d'observer des phénomènes résultant de l'action éolienne dans quatre milieux géologiques bien distincts: les plaines alluviales; les terrasses alluviales; les épandages fluvio-glaciaires; les formations d'âge mésozoïque et d'âge tertiaire.

\section{Plaines alluviales}

$\mathrm{Au}$ cours de périodes d'étiage en été, dans les régions où le lit des cours d'eau à chenaux anastomosés comprend des sédiments à granulométrie fine, il est possible pour le vent de prendre en charge des sédiments. Le 17 juillet 1975 le long du cours inférieur de la rivière Thomsen et le long de la rivière Desert qui s'écoule vers le nord à une dizaine de kilomètres à l'ouest de la baie Castel (fig. 3D), des nuages de poussières s'élevant sur une distance de plusieurs dizaines de mètres au-dessus de la plaine alluviale ont été observés. Des mesures à l'aide d'un anémomètre, ont révélé que les vents qui venaient alors du nord, avaient une vitesse moyenne de $22.4 \mathrm{~km} / \mathrm{h}$. Il est donc intéressant de noter que sur l'île de Banks, des vents relativement faibles trouvent parfois dans les plaines alluviales où l'on observe de larges lits fluviatiles à chenaux anastomosés partiellement asséchés en surface et sur lesquels la couverture caillouteuse est localement tout à fait absente, des conditions propices au transport de silt et de sable. Cette action éolienne se pro- 
duit également l'hiver comme le prouvent les nombreux dépôts sableux interstratifiés au milieu des bancs de neige qui ont été observés près de la rivière Thomsen (voir photo 10.2, dans French (1976)).

\section{Terrasses alluviales}

Les résultats de l'action éolienne sur l'île de Banks s'observent le plus fréquemment à la surface de terrasses situées le long des principaux cours d'eau tels la rivière Bernard (fig. 3C) et la rivière Thomsen (figs $3 \mathrm{D}$ et $3 \mathrm{E}$ ). Sur ces terrasses s'accumulent les sédiments emportés depuis les plaines alluviales et s'exercent en même temps des processus de déflation et de dépôt des sédiments éoliens plus anciens. Il existe une relation étroite entre la localisation de cette activité éolienne et la localisation de certaines formations géologiques. De part et d'autre de la rivière Thomsen (figs 3D et 3E), la formation d'Isachsen du Crétacé supérieur, localement composée de sables quartzitiques, affleure entre les latitudes $73^{\circ} 25^{\prime} \mathrm{N}$ et $73^{\circ} 53^{\prime} \mathrm{N}$. Or, la plus grande partie de l'activité éolienne observée à proximité de la rivière Thomsen se produit précisément entre ces latitudes. Comme nous le verrons plus tard, les sables des accumulations éoliennes de cette région proviennent en effet essentiellement de cette formation d'Isachsen. Le même lien existe également dans la région au nord de la baie $\mathrm{De}$ Salis (fig. 3B). Là, des alluvions provenant de l'érosion fluviale de sables d'Isachsen sont remaniées par le vent. Dans la région au sud du coude de la rivière Bernard (fig. 3C) l'actıvité éolienne semble être liée directement à la présence de sables de la formation de Beaufort du Néogène. A cet endroit, la rivière s'est encaissée dans les sables et graviers de cette formation en produisant des alluvions sableuses qui ont été remaniées par le vent.

\section{Epandages fluvio-glaciaires}

A la surface d'anciennes plaines d'épandages fluvio-glaciaires sises à proximité de la limite de la dernière avancée glaciaire d'âge wisconsinien, dans le cours supérieur de la rivière Thomsen (fig. 3E) et dans la région au sud-est de Sachs Harbour (fig. 3A), la déflation est intense et souvent spectaculaire. La déflation agit sur des sables éoliens qui proviennent à l'origine du remaniement de sables fluvio-glaciaires, probablement au moment de la formation des plaines d'épandages. Souvent, sur ces zones de déflation, les sables sont fixés entre autres par le saule Salix alaxensis, donnant ainsi naissance à toute une série de buttes résiduelles, qui atteignent jusqu'à $3 \mathrm{~m}$ de hauteur, et sont séparées par des creux de déflation (fig. 5).

\section{Formations d'âge mésozoïque et tertiaire}

Les processus éoliens sont également actifs aux endroits où des sédiments non consolidés, à texture fine comme des sables fins et des silts d'âge mésozoïque et tertiaire, affleurent en surface. Durant les saisons d'été, ces sédiments bien triés peuvent facilement être érodés et transportés par le vent surtout lorsque le sédiment est bien asséché et le tapis végétal restreint.

Dans la région à l'ouest de la baie Castel (fig. 3D), des étendues de sables fins et silts grossiers, dont plus de $90 \%$ de la masse totale se situe entre $250 \mu \mathrm{m}$ et $31 \mu \mathrm{m}$, de la formation d'Eureka Sound du Paléogène, sont ainsi modifiés par le vent. A deux reprises au cours de l'été 1974 et de l'été 1975, des nuages de poussières ont été observés dans cette région. Localement des zones de déflation ainsi que des accumulations éoliennes ont été observées. L'action éolienne est importante dans les régions où les sédiments de cette formation ne sont pas fixés par la végétation. D'autre part, vu le tri excellent de ces dépôts, il ne se produit pas de concentration d'éléments grossiers en surface qui serait susceptible de bloquer la déflation.

En plusieurs endroits, le long de la rivière Thomsen, le même genre d'action éolienne a été noté dans les sables bien triés de la formation d'Isachen. Enfin, il semble également que la corrasion éolienne joue un rôle dans le façonnement de certaines surfaces structurales. Ainsi, par exemple, dans une région de 'badlands' le long de la rivière Muskox, le vent met en relief des lits alternants de sables fins et de sédiments organiques de la formation d'Eureka Sound.

\section{Autres domaines d'action éolienne}

En de nombreux endroits et dans diverses unités lithologiques, on retrouve en surface un placage mince et continu d'éléments grossiers d'un diamètre de plus de $40 \mathrm{~mm}$ et ce, sur des unités qui ne recèlent qu'une faible proportion de ces éléments dans leur composition originale. Il est difficile d'évaluer quelle est la part, dans la formation de ces nappes d'éléments résiduels, qui peut être imputée respectivement au vent, au ruissellement et à la migration des cailloux vers la surface sous l'action de gel. Une chose est cependant très probable, c'est que le vent a joué 
un rôle dans l'évacuation des débris fins. Bird (1967, pp. 120, 240 et 273) a d'ailleurs affimé, avant nous, que le vent est responsable de la concentration en surface d'éléments grossiers en plusieurs endroits de l'Arctique, particulièrement sur les deltas soulevés, les terrasses et les eskers.

\section{La couverture végétale des sédiments éoliens}

La plupart des espèces reconnues par Kuc (1974), Mason et al. (1972), et, von Thannheiser et Schweingruber (1974) sur l'île de Banks existent sur les surfaces soumises aux actions éoliennes. Toutefois, les régions recouvertes par des sédiments éoliens présentent une grande diversité dans le pourcentage de couverture des divers peuplements végétaux. Sous cet aspect, elles peuvent être groupées en trois principaux domaines: (1) les surfaces actives où l'érosion et l'accumulation éolienne est importante; (2) les surfaces partiellement stabilisées où la couverture végétale est clairsemée ou peu dense et caractérisée par des monticules de sédiments éoliens recouverts de végétation; (3) les surfaces
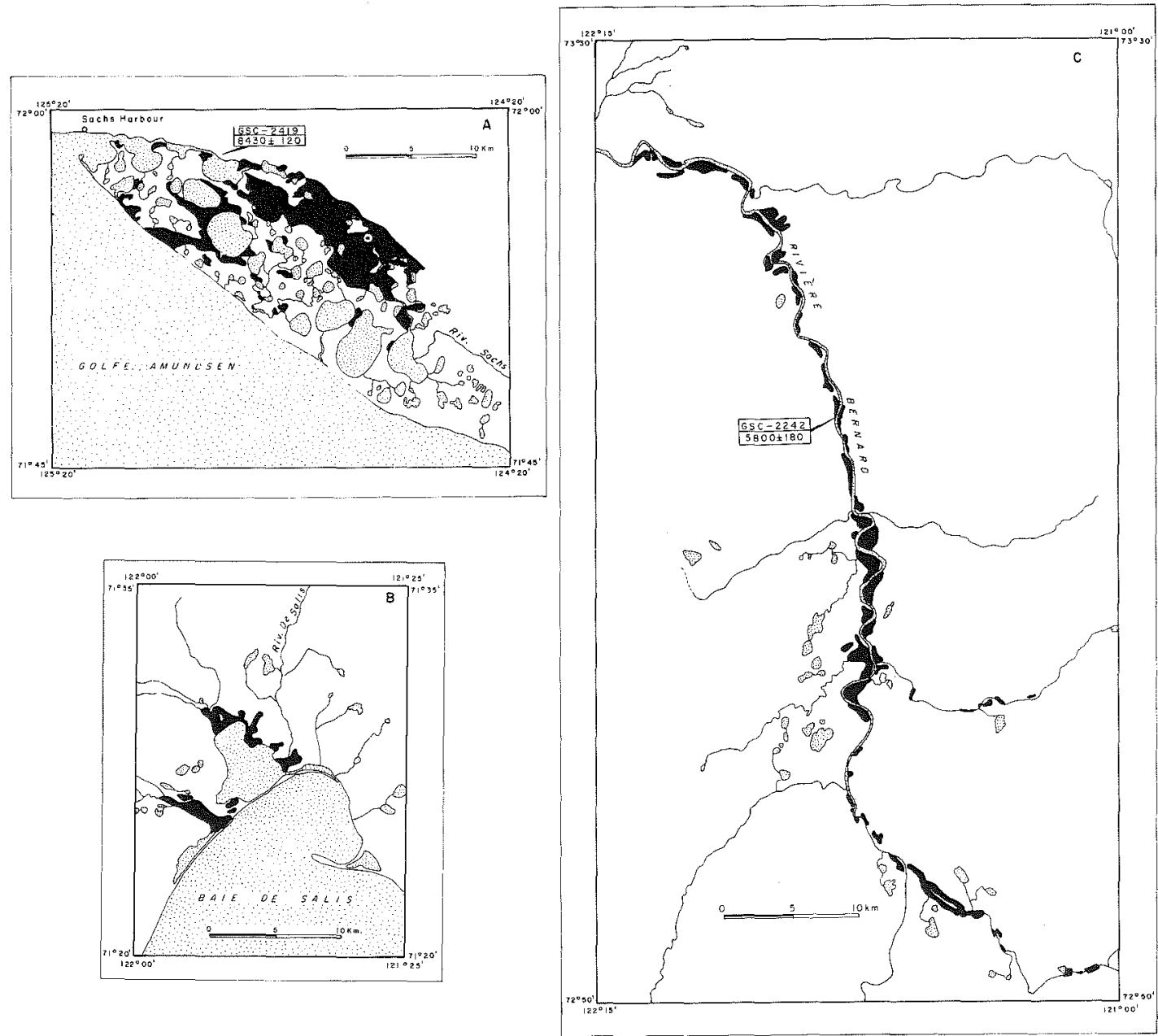

FIG. 3. Cartes montrant la répartition de toutes les régions où l'action éolienne est intense sur l'île de Banks. Ces localités sont représentées par des taches noires. Les cartes montrent également la localisation de certains sites mentionnés dans le texte ainsi que le lieu de prélèvement des échantillons datés au ${ }^{14} \mathrm{C}$. (A) Région au sud-est de Sachs Harbour. (B) Région au nord de la baie de Salis. (C) Région au sud du coude de la rivière Bernard. (D) Région du cours inférieure de la rivière Thomsen. (E) Région du cours supérieure de la rivière Thomsen. 

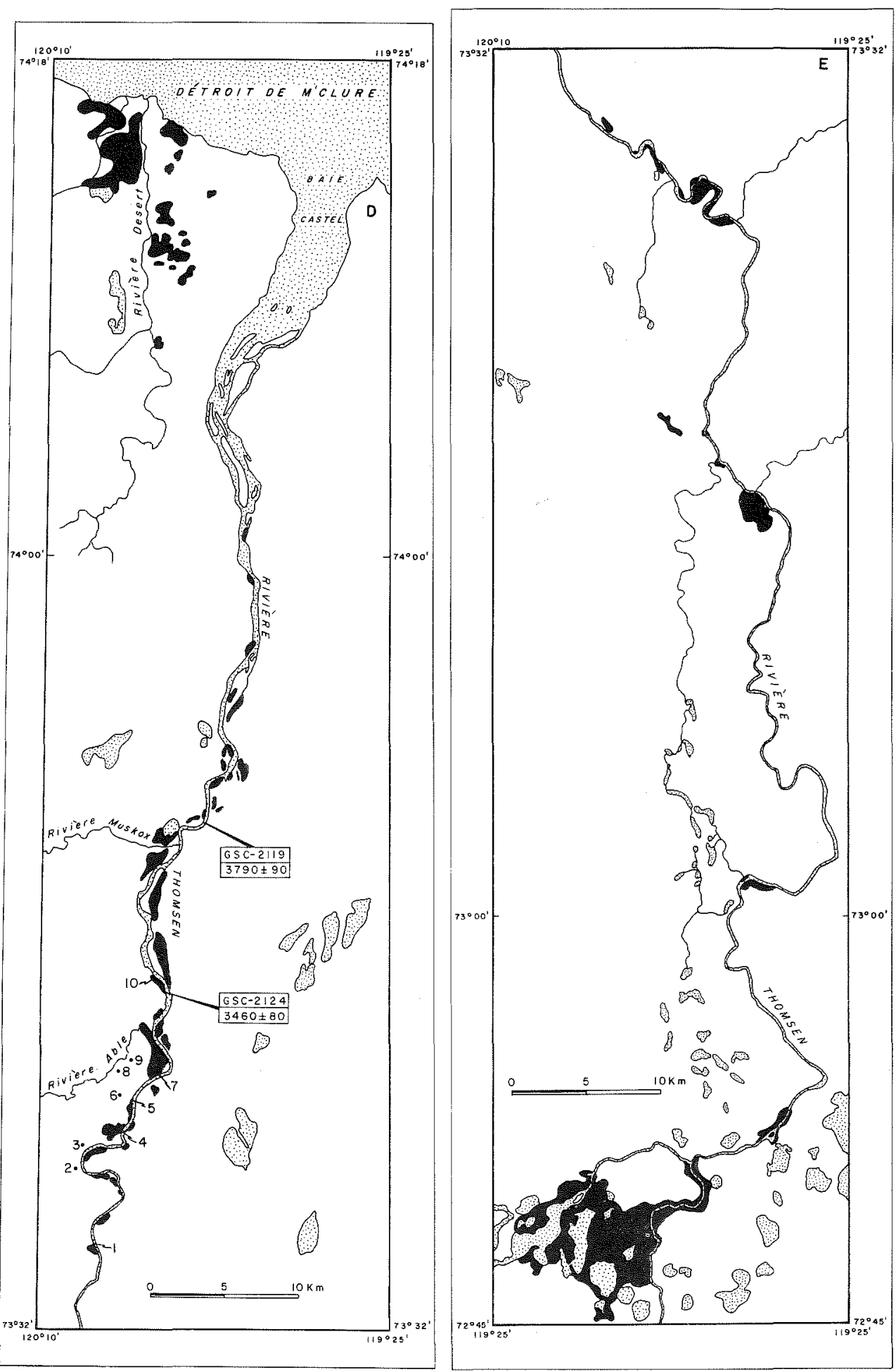


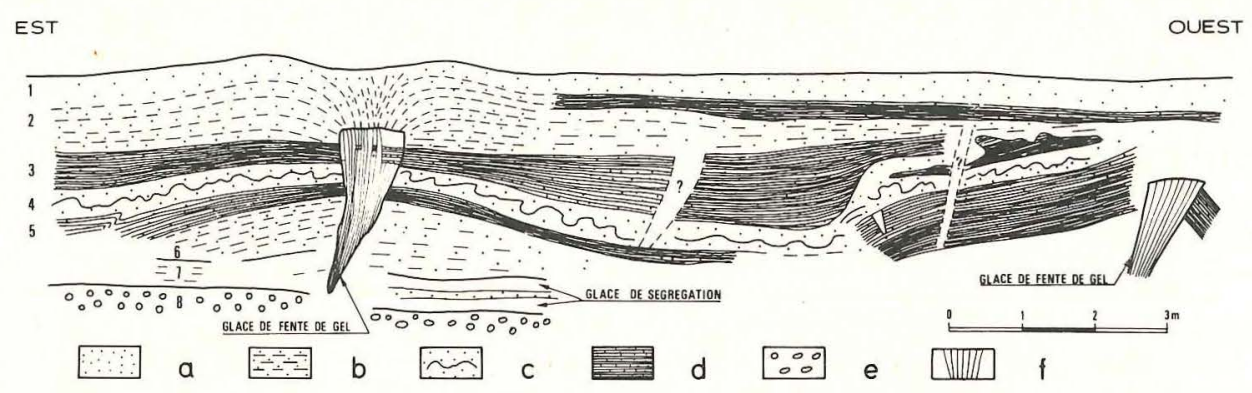

Fig. 4. Coupe dégagée dans des sables éoliens plus ou moins tourbeux reposant sur un cailloutis fluviatile à proximité de la rivière Thomsen (site 4 de la fig. 3D). L'échelle des hauteurs n'est pas exagérée. Une photo de la fente de gel située à la partie est de la coupe est donnée sur la fig. 9. (a) sables fins, non lités; (b) alternance de lits de sables fins et de sables grossiers, avec rares lits humifères; (c) sables fins et moyens avec des lits de matières organiques cryoturbés; (d) tourbe sableuse; (e) graviers fluviatiles; (f) glace de fente de gel.

de sédimentation éolienne stabilisée à couverture végétale dense.

Le tableau 2, préparé par le troisième auteur présente les espèces que l'on retrouve dans ces divers domaines.

\section{Les surfaces actives}

Les surfaces actives sont démunies de végétation. Les sédiments qui s'y trouvent sont très mobiles et ne constituent pas un substratum stable permettant une colonisation végétale.

\section{Surfaces à stabilité partielle}

On trouve, à proximité des milieux actifs, des secteurs partiellement colonisés par des plantes vasculaires et ceci malgré une érosion et une accumulation éolienne assez efficace. La multiplication végétale se fait par la croissance de rhizomes courts, ce qui donne des tiges disposées en touffes serrées. Ici, la forme typique de croissance des plantes vasculaires, telles que les herbes et différentes plantes herbacées, est la forme en touffe compacte, encouragée par l'érosion éolienne du sable autour des racines et par la mise en place de sable du côté abrité de la plante. Il arrive souvent que des plantes qui n'adoptent pas en général la forme de touffe, la prennent dans une localité où l'action éolienne est partiellement active. Le buisson Salix alaxensis forme des monticules parfois spectaculaires dont quelques-uns mesurent plusieurs mètres de diamètre (fig. 5).

Les surfaces à stabilité partielle offrent des niches particulières où des espèces que l'on trouve rarement sur l'île de Banks et les îles de l'Arctique poussent en abondance. Leur diversité est particulièrement marquée dans la région de la rivière Sachs (fig. 2A) où l'érosion éolienne est importante. On peut également observer certaines de ces plantes rares sur d'autres surfaces éoliennes de l'île de Banks. Les dépôts éoliens dans les régions plus élevées présentent moins de diversité.

\section{Les surfaces stables}

Les surfaces éoliennes stables se trouvent dans des milieux de sédimentation éolienne, souvent à proximité de surfaces hautement actives. Plus de $75 \%$ de la surface de ces régions sont couvertes de végétation et se trouvent en général sur des pentes douces reliées à des dépressions de faible profondeur, incluant d'anciens bassins lacustres, et sur d'anciennes terrasses. De petites mares ayant une origine thermokarstique se rencontrent souvent dans ce secteur.

On trouve deux types d'associations végétales dans ces régions stabilisées: les communautés mésophytes sur les surfaces moyennement drainées, et des communautés hydriques dans les localités mal drainées ou parsemées de mares.

Il existe deux genres de communauté mésophytes qui ont toutes deux des buissons comme plantes vasculaires dominantes. Le long de la rivière Sachs, la communauté de buissons Salix arctica - Dryas integrifolia, et la communauté de buissons et fourrés Salix alaxensis - Salix richardsonii se trouvent autour des lacs et dans les zones où l'on observe des congères de neige tardive. Dans les autres zones éoliennes de l'île de Banks, la communauté de buissons nains domine les endroits mésophytes.

La communauté de buissons et fourrés $S$. alaxensis $-S$. richardsonii peut être observée dans les endroits où une forte accumulation de 


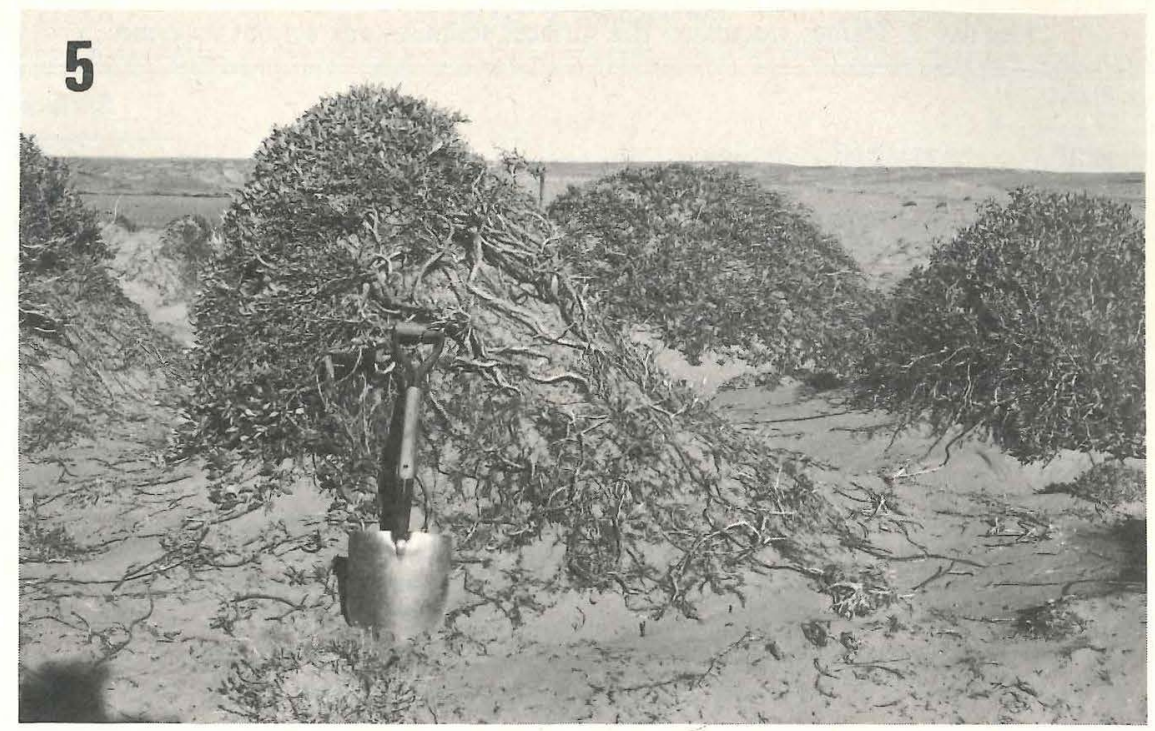

FIG. 5. Buttes résiduelles fixées par des saules et séparées par des creux de déflation, sur la plaine d'épandage fluvio-glaciaire au sud-est de Sachs Harbour (cliché no 202927 de la Commission géologique du Canada).

neige protège les longues tiges. Il existe peu d'associés vasculaires, sauf quelques Arctostaphylos rubra dans les fourrés plus clairsemés, et quelques touffes d'herbe. On trouve parfois une couche inférieure dense de cryptogames qu'on ne recontre pas dans les zones où il $\mathrm{y}$ a un mouvement de sables en surface.

La communauté de buissons nains (SalixDryas) sur les sites mésophytes présente une couche inférieure dense de plantes vasculaires comme entre autres Alopecurus alpinus, Kobresia sp., Carex rupestris et Luzula confusa.

Sur les pentes mal drainées, et autour des mares et lacs, on rencontre plusieurs communautés de laîches. Une couche dense de mousse (souvent Drepanocladus sp.) forme la couche inférieure. Carex stans, accompagné souvent du Salix arctica, se trouve en abondance dans les zones où la présence d'eau en surface est très rare. On trouve Salix sur des tertres mousseux et dans des micro-habitats plus secs au sein de la communauté. Carex stans, et parfois Carex bigelowii et Carex membranacea, et de l'herbe tomenteuse Eriophorum angustifolium et Eriophorum scheuchzeri sont présents sur le bord des mares et les endroits mal drainés. Dans certains endroits, on trouve des groupes denses d'herbes comme Dupontia fisheri et Alopecurus alpinus mais elles sont en général distribuées de façon clairsemée dans les communautés de laîches.
Quelques fois, on trouve des couches de Drepanocladus dans lesquelles se perdent quelques plantes vasculaires, en général des touffes d'Alopecurus sp. de Dupontia sp. et de Ranunculus hyperboreus. Ces sites sont souvent des mares complètement comblées de mousses.

$\mathrm{Au}$ centre et le long des berges de petites mares peu profondes, Pleuropogon sabinei et localement Hippurus vulgaris et Arctophila fulva émergent en surface.

Bien que ces milieux soient actuellement stables, ils peuvent devenir actifs si, par une modification de la couverture végétale, les sédiments non consolidés sus-jacents sont exposés à l'action éolienne.

\section{Les dépôts éoliens de la vallée de la Thomsen}

Une des localités où l'action éolienne est importante se situe dans le fond de la vallée de la rivière Thomsen, de part et d'autre de sa confluence avec la rivière Able (fig. 3D). Nous décrivons ci-dessous, à titre d'exemple, les dépôts éoliens qui s'y trouvent.

\section{Morphologie et couverture végétale des zones de déflation}

Sur la terrasse inférieure de la rivière Thomsen dans la région mentionnée ci-dessus, l'action éolienne est parfois évidente comme le montrent les figs 6 et 7 (site 7 de la fig. 3D). L'importance de l'érosion éolienne est souvent attestée par des 
TABLEAU 2. Plantes vasculaires des surfaces soumises aux actions éoliennes

\begin{tabular}{|c|c|c|c|c|}
\hline \multirow{2}{*}{\multicolumn{2}{|c|}{ Plantes vasculaires }} & \multirow[b]{2}{*}{$\begin{array}{l}\text { Surfaces à } \\
\text { stabilité } \\
\text { partielle }\end{array}$} & \multicolumn{2}{|c|}{ Surfaces stables } \\
\hline & & & $\begin{array}{c}\text { Sites } \\
\text { moyennement } \\
\text { bien drainés }\end{array}$ & $\begin{array}{l}\text { Sites mal } \\
\text { drainés }\end{array}$ \\
\hline \multirow{31}{*}{ Graminées et formes similaires } & Equisetaceae & & & \\
\hline & $\begin{array}{l}\text { Equisetum arvense } \mathrm{L} . \\
\text { E. variegatum Schleich. }\end{array}$ & + & & + \\
\hline & Graminae & & & \\
\hline & $\begin{array}{l}{ }^{*} \text { Hierochloe alpina } \mathrm{R} \text {. et } \mathrm{S} \text {. } \\
{ }^{*} H \text { paucifora } \mathrm{R} \mathrm{Br}\end{array}$ & $\begin{array}{l}+ \\
+\end{array}$ & & \\
\hline & *Alopecurus alpinus $\mathrm{L}$. & $\begin{array}{l}+ \\
+\end{array}$ & + & + \\
\hline & * Deschampsia sp. & + & & \\
\hline & *Trisetum spicatum & + & & \\
\hline & $\begin{array}{l}\text { *Poa alpigena Lindm. var. } \\
\text { vivipara Schol. }\end{array}$ & + & & \\
\hline & P. arctica $\mathrm{R}$. Br. & + & & \\
\hline & P. abbreviata $\mathrm{R} . \mathrm{Br}$ & + & & \\
\hline & $\begin{array}{l}\text { Pleuropogon Sabinei R. Br. } \\
{ }^{2} \text { Arctophila fulva Anders }\end{array}$ & & & + \\
\hline & & & & $\begin{array}{l}+ \\
+\end{array}$ \\
\hline & Puccinellia sp. & + & & \\
\hline & $\begin{array}{l}\text { Festuca baffinensis Polunin } \\
\text { F. rubra L. }\end{array}$ & + & & \\
\hline & * Agropyron latiglume $\mathrm{Rydb}$ & + & + & \\
\hline & ${ }^{* 2}$ Elymus arenarius L. ssp. mollis. & + & & \\
\hline & $\begin{array}{l}\text { Hult. } \\
\text { Cyperaceae }\end{array}$ & & & \\
\hline & $\begin{array}{l}\text { Cyperaceae } \\
\text { Eriophorum angustifolium Honck. }\end{array}$ & & & $t$ \\
\hline & E. scheuchzeri Hoppe & & & $\begin{array}{l}+ \\
+\end{array}$ \\
\hline & * Kobresia sp. & + & + & \\
\hline & Carex nardina $\mathrm{Fr}$. & & + & \\
\hline & C. scirpoidea Michx. & & + & \\
\hline & C. rupestris All. & & + & \\
\hline & C. maritima Gunn. & + & & \\
\hline & ${ }^{*}$ C. Bigelowii Torr. & & + & + \\
\hline & C. stans Drej & & & + \\
\hline & C. membranaceae Hook. & & & + \\
\hline & Juncaceae & & & \\
\hline & Juncus biglumis $\mathrm{L}$. & & & + \\
\hline & ${ }^{*}$ Luzula confusa Lindeb. & & + & + \\
\hline & Polygonaceae & & & \\
\hline \multirow[t]{21}{*}{ Herbes } & *Oxyria digyna Hill & + & + & \\
\hline & Polygonum viviparum $\mathrm{L}$. & & + & + \\
\hline & Caryophyllaceae & & & \\
\hline & Stellaria sp. & + & + & \\
\hline & Cerastium sp. & + & + & \\
\hline & Cerastium regelii Ostf. & & & + \\
\hline & Arenaria sp. & + & + & \\
\hline & Silene acaulis $\mathrm{L}$. & + & + & \\
\hline & Melandrum apetalum Fenzl. & & + & + \\
\hline & Ranunculaceae & & & \\
\hline & Ranunculus sp. & & + & + \\
\hline & Pulsatilla ludoviciana (Nutt.) Heller & + & & \\
\hline & Papaveraceae & & & \\
\hline & *Papaver radicatum Rottb. & + & + & \\
\hline & $\begin{array}{l}\text { Cruciterae } \\
\text { Cochlearia officinalis } \mathbf{L}\end{array}$ & + & & \\
\hline & ${ }^{3}$ Descurainia sophioides Schulz & + & & \\
\hline & Cruciferae & & & \\
\hline & Lesquerella arctica Wats. & + & & \\
\hline & Draba sp. & + & + & \\
\hline & Erysimum pallasii Fern. & + & & \\
\hline & Braya purpurascens Bunge & + & & \\
\hline
\end{tabular}


TABleau 2. (Suite et fin)

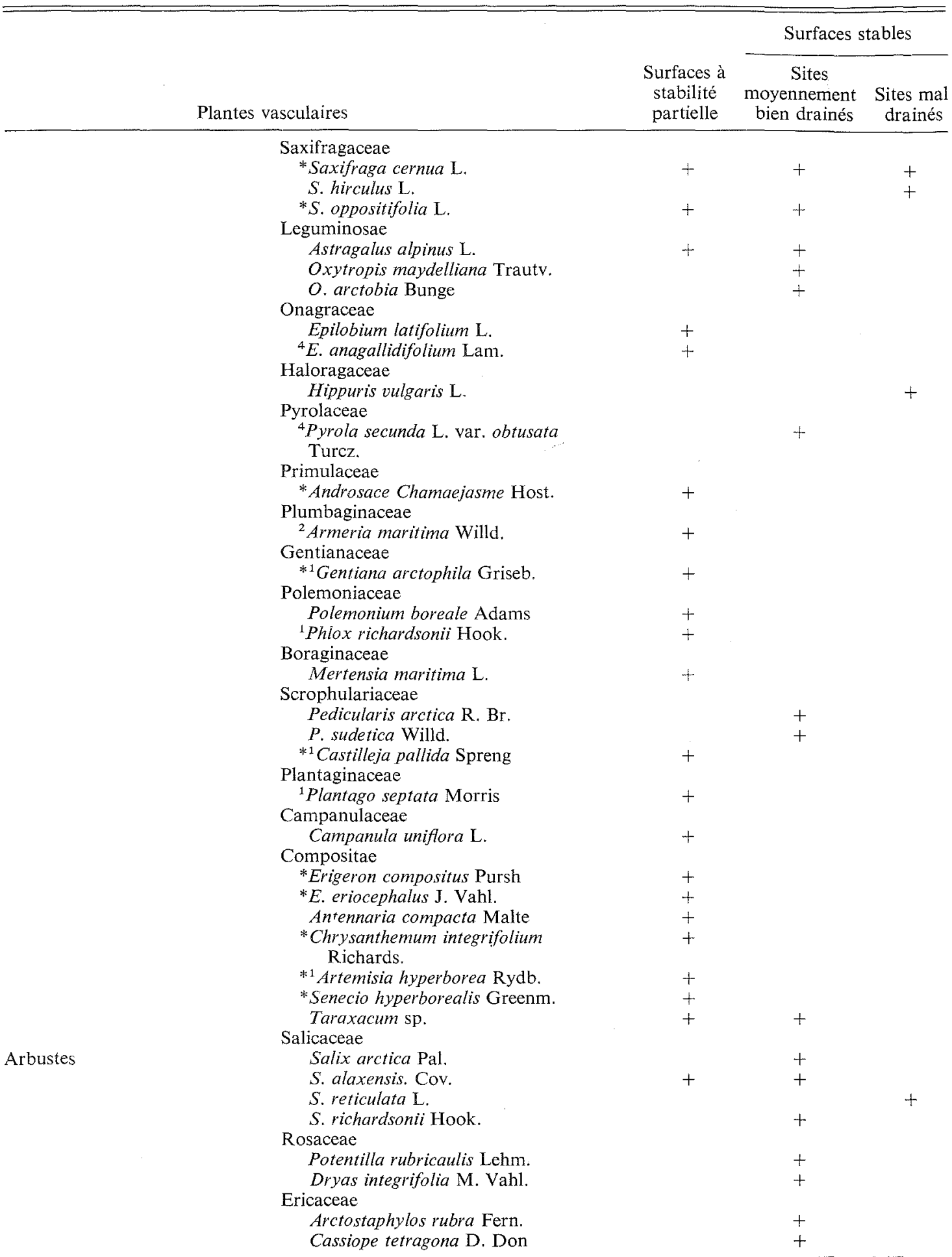

${ }_{1}^{1}$ Plantes vasculaires endémiques au secteur continental de la région arctique nord-américaine.

2 Espèces du bas-arctique.

${ }^{3}$ Espèces asiatiques.

${ }^{4}$ Espèces subarctiques et alpines.

* Plantes vasculaires dont la forme est en touffe. 


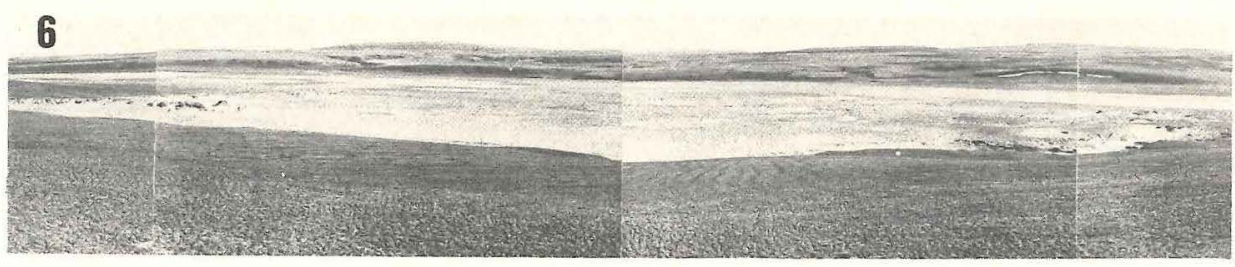

FIG. 6. Vue panoramique vers l'est d'un site sur la rive gauche de la rivière Thomsen où l'action éolienne est actuellement très active. Photographie prise en direction de l'est à la localité 7 de la fig. $3 \mathrm{D}$; le nord est à gauche, le sud à droite de cette photo.

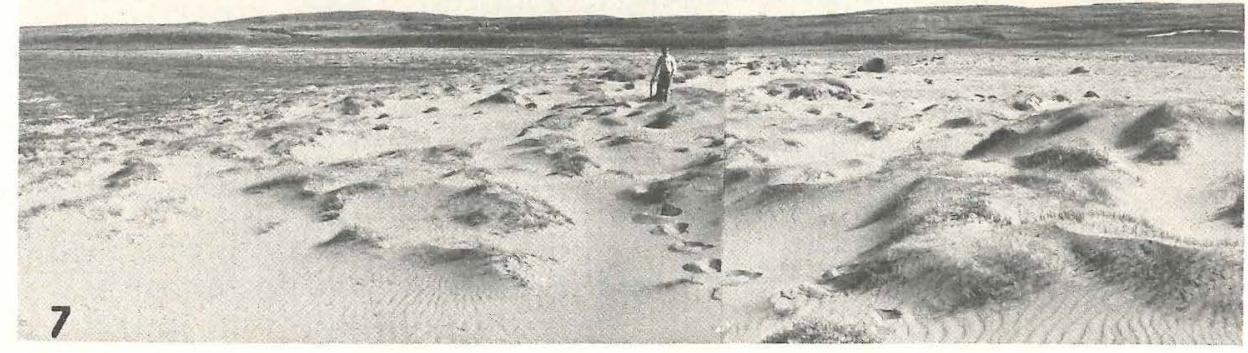

FIG. 7. Accumulation de sables éoliens sur la rive gauche de la rivière Thomsen dans la zone visible à l'extrémité gauche de la fig. 5 .

buttes témoins de la surface originelle, terrains toujours fixés par la végétation et qui dominent les creux de déflation. Cette morphologie n'existe cependant que sur des dépôts éoliens précédemment fixés, qui sont actuellement remis en mouvement.

Les formes d'accumulation éolienne sont peu marquées, accumulation derrière une touffe végétale par exemple, et il est assez exceptionnel de trouver une accumulation semblable à celle que montre la fig. 7 ou celle qui a été observée au site 1 de la fig. 3D. En cet endroit, des accumulations de sables éoliens sont en train de combler la vallée d'un affluent de la rivière Thomsen provoquant le déplacement du chenal de l'affluent.

Généralement près des zones actives de déflation, le sable est mis en place dans la maigre couverture végétale qui le fixe et le dissimule. A partir de ce lent processus d'accumulation, il se produit avec le temps, des séquences considérables de sédiments éoliens comme en témoigne la coupe que nous décrivons plus loin.

Localement des traînées sableuses existent sur la végétation herbacée de la toundra. Ces sables paraissent avoir été mis en place sur la couverture neigeuse. La sédimentation éolienne en hiver est attestée par des dépôts nivéo-éoliens, apparaissant comme des couches de sables alternant avec des couches de neige. Le litage de ces dépôts est toutefois détruit l'été, la fusion de la neige déterminant un bouleversement et un mélange complet des particules minérales qui donne ces traînées sableuses.

A la localité 5 indiquée sur la fig. 3D, un cailloutis de basse terrasse situé environ $2 \mathrm{~m}$ audessus du niveau des plus hautes eaux affleure entre la rivière et des dépôts éoliens. Plusieurs cailloux éolisés y ont été observés. Ce façonnement est évident sur des roches à grain fin telles que le quartzite et le diabase et apparaît par un poli caractéristique, des arêtes éoliennes et même parfois, par des cupules; toutefois, celles-ci sont peut convaincantes. Sur certains cailloux éolisés avaient grandi des lichens. Ceux-ci étaient cependant totalement absents sur les faces exposées au sud-est, indiquant une action actuelle des vents soufflant depuis cette direction.

\section{Les dépôts éoliens vus en coupe}

La déflation éolienne s'exerce le long de la rivière Thomsen au détriment de formations éoliennes stabilisées, généralement épaisses de plusieurs mètres. Il s'agit de dépôts sableux stratifiés formés de couches de quelques millimètres à $2 \mathrm{~cm}$ d'épaisseur, ordinairement peu régulières et soulignées par des horizons humifères (fig. 8). Au sein de ces dépôts, des racines et des branches de saule nain sont conservées. D'un centimètre à l'autre existent des variations granulomètriques importantes s'étendant des 


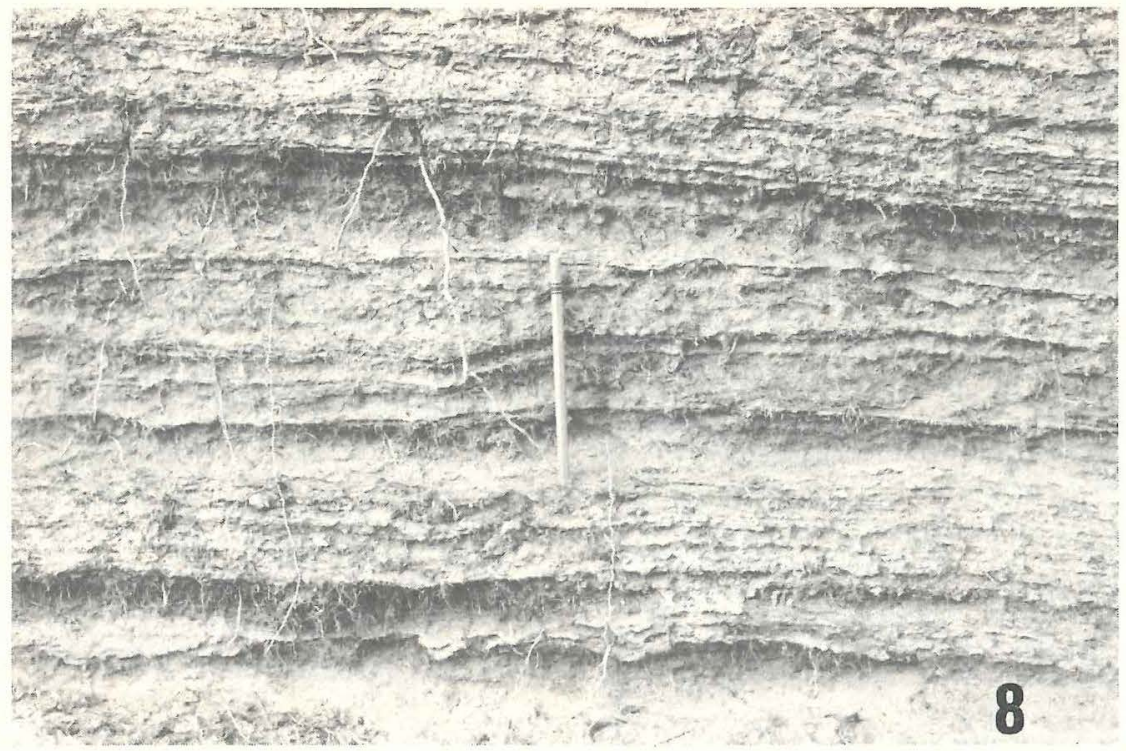

FIG. 8. Coupe à proximité de l'embouchure de la rivière Muskox dans la Thomsen, dans des dépôts éoliens montrant des couches alternantes de sables éoliens fins et d'horizons humifères. Le crayon servant d'échelle mesure $15 \mathrm{~cm}$ (cliché no 202926 de la Commission géologique du Canada).

silts jusqu'aux granules de $2 \mathrm{~mm}$ de diamètre. La stratification est généralement horizontale et une même couche peut être suivie sur plusieurs mètres de longueur. Le litage est parfois interrompu par de fines fentes obliques, sans doute des fentes de gel, qui se succèdent avec un espacement de 2 à 5 ini.

Ces formations sableuses témoignent d'une sédimentation éolienne lente, semblable à celle que nous observons aujourd'hui sur la faible couverture végétale de la toundra, en bordure des zones où s'exerce la déflation éolienne. Elles attestent de la progression synchrone de la couverture végétale en compétition avec le taux d'accumulation des sédiments.

Ces dépôts sableux ont localement une épaisseur considérable que l'on ne peut mesurer qu'en des endroits où ils sont incisés par un ravinement récent. Des épaisseurs de 6 à $7 \mathrm{~m}$ ont été notées en plusieurs endroits.

Une coupe a été dégagée à l'endroit 4 de la fig. 3D en rafraîchissant la paroi d'un ravin. Elle nous a montré au-dessus d'une basse terrasse de la Thomsen située environ $2.5 \mathrm{~m}$ au-dessus du niveau des hautes eaux de la rivière, $280 \mathrm{~cm}$ de sables éoliens tourbeux (fig. 4). En surface, un réseau de dépressions peu profondes trahissait l'existence de fentes de gel. La séquence observée témoigne d'une évolution relativement complexe contrôlée, comme nous allons le montrer, par l'apparition de fentes de gel.

Deux fentes de gel à remplissage de glace ont été observées. Celle qui est située à l'extrémité ouest de la coupe paraît actuellement inactive et l'accumulation de sables éoliens en a effacé toute trace en surface. Par contre, la fente de gel visible à l'est de la coupe paraît active et d'après la morphologie de la surface, elle semble s'ouvrir chaque année. Elle présente cependant une particularité remarquable (fig. 9). Alors que sa partie inférieure est constituée de glace au sein de laquelle les inciusions minérales dessinent, comme dans toutes les fissures à fente de gel caractéristiques, une zonation verticale, la partie supérieure montre, localement, une zonation horizontale due à l'alternance de couches minérales et de bandes de glace. Cette glace est de la glace de ségrégation qui a grandi au-dessus de la glace de fente de gel (Katasonov 1967; Mackay 1972). C'est une conséquence de l'élévation du pergélisol à la suite de l'accumulation de sable éolien en surface. Ces lentilles de glace de ségrégation témoignent que cette fente de gel est syngénétique du dépôt, c'est-à-dire qu'elle s'est accrue vers la surface malgré sa faible dimension, au fur et à mesure de l'accumulation des sables éoliens. La présence possible de ce type de glace sur l'île de Banks, dénommée “aggrada- 


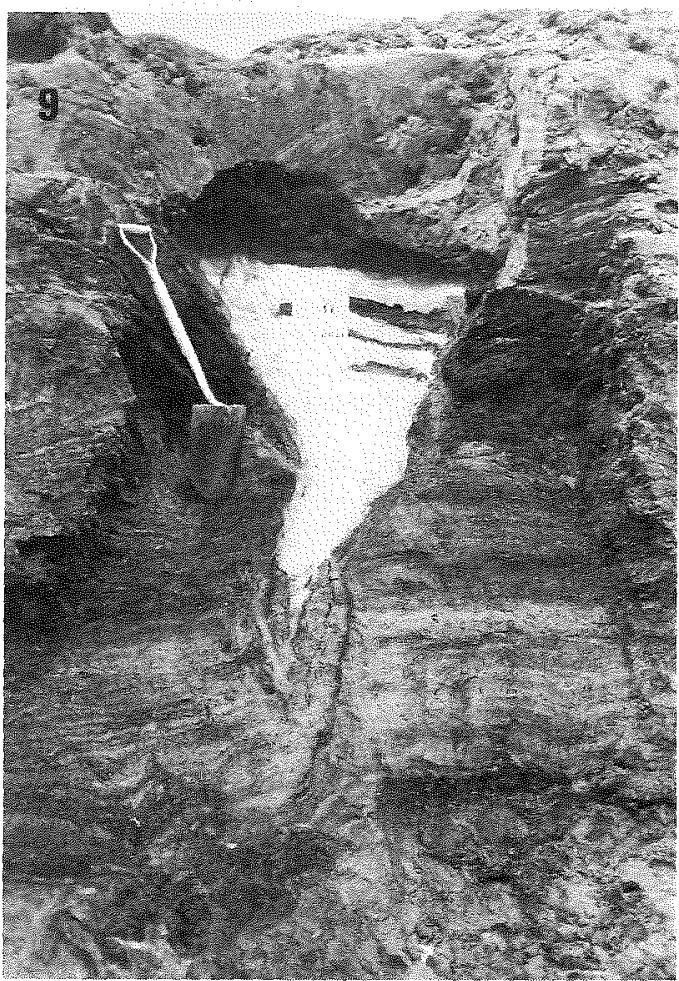

Fig. 9. Photo d'une fente de gel développée dans des sables éoliens de l'île de Banks près de la rivière Thomsen. Cette fente de gel est dessinée sur la fig. 4. A la partie supérieure droite du coin de glace apparaissent 3 lentilles de glace de ségrégation, séparées par des couches minérales horizontales. Cette glace de ségrégation témoigne de l'élévation du pergélisol suite à l'accumulation des sables éoliens.

tional ice" par Mackay (1972), a déjà été signalée par French et Egginton (1973).

$\mathrm{Vu}$ l'importance générale de ce processus, nous détaillons ci-dessous comment ce phénomène a dû se produire (voir également Pissart (1975)). L'apparition de glace de ségrégation nécessite: (a) un gel lent, (b) la présence d'humidité, (c) une granulométrie fine. Ces conditions sont parfaitement remplies lorsque, à la suite d'une sédimentation lente, la surface du sol s'élève progressivement en étant suivie par le sommet du pergélisol. Au moment où commence chaque hiver, la reprise de li zone active par le gel s'effectue non seulement de la surface vers la profondeur, mais aussi, simultanément depuis le sommet du pergélisol vers le haut. Dans cette dernière direction, la progression du gel est évidemment fort lente. Comme d'autre part, l'humidité au somment du pergélisol est souvent considérable, si la granulométrie est favorable, des lentilles de glace de ségrégation caractéristiques peuvent $y$ apparaître. Elles n'y seront conservées que si le sommet du pergélisol s'élève, c'est-à-dire si elles ne fondent pas au cours de l'été suivant. Cette élévation peut résulter d'une sédimentation continue à la surface, d'un refroidissement climatique, ou encore d'une modification dans les conditions externes telles que l'humidité, la couverture végétale ou d'autres, qui contrôlent le développement du pergélisol. Ailleurs, la glace de ségrégation apparue est comprise dans la zone active et doit sans doute disparaître et réapparaître chaque année.

C'est par ce mécanisme que s'expliquent également les lentilles de glace de ségrégation visibles sur la même coupe (fig. 4) au-dessus du gravier, à $2.50 \mathrm{~m}$ de profondeur. Elles ont dû apparaître au cours de la sédimentation éolienne, à un moment où existait déjà un pergélisol profond.

Entre les deux coins de glace, les dépôts témoignent du développement de polygones de fente de gel à centre déprimé. En effet, une couche de tourbe sableuse (fig. 4, couche no 3) atteint localement une épaisseur de $1.20 \mathrm{~m}$ alors que latéralement, elle ne dépasse pas $20 \mathrm{~cm}$. Cette accumulation de tourbe sableuse résulte du colmatage d'une cuvette apparue entre les deux coins de glace. En effet, dans les régions voisines, des cuvettes semblables existent. Elles apparaissent et évoluent de la façon suivante: au début, les cuvettes apparaissent par la formation de bourrelets au-dessus de fentes de gel; elles s'approfondissent ensuite par des phénomènes thermokarstiques, c'est-à-dire par fusion de glace, sans doute de glace de ségrégation formée, comme nous l'avons vu plus haut, dans les sables sous-jacents. La fusion est provoquée par la formation d'une mare qui en déterminant le réchauffement du sol, s'approfondit rapidement. La cuvette inondée se colmate ensuite de mousses et de sables éoliens en donnant la tourbe sableuse que nous observons.

Le stade ultime de l'évolution peut être provoqué par une reprise de la déflation dénudant la surface au-dessus des fentes de gel et déclenchant leur fusion. Des ravins en résultent qui draînent les cuvettes thermokarstiques et transportent vers la rivière des quantités importantes de sable.

Sans modification climatique, les phénomènes mentionnés ci-dessous ont donc pu se produire: accumulation de sables éoliens, soulèvement du 


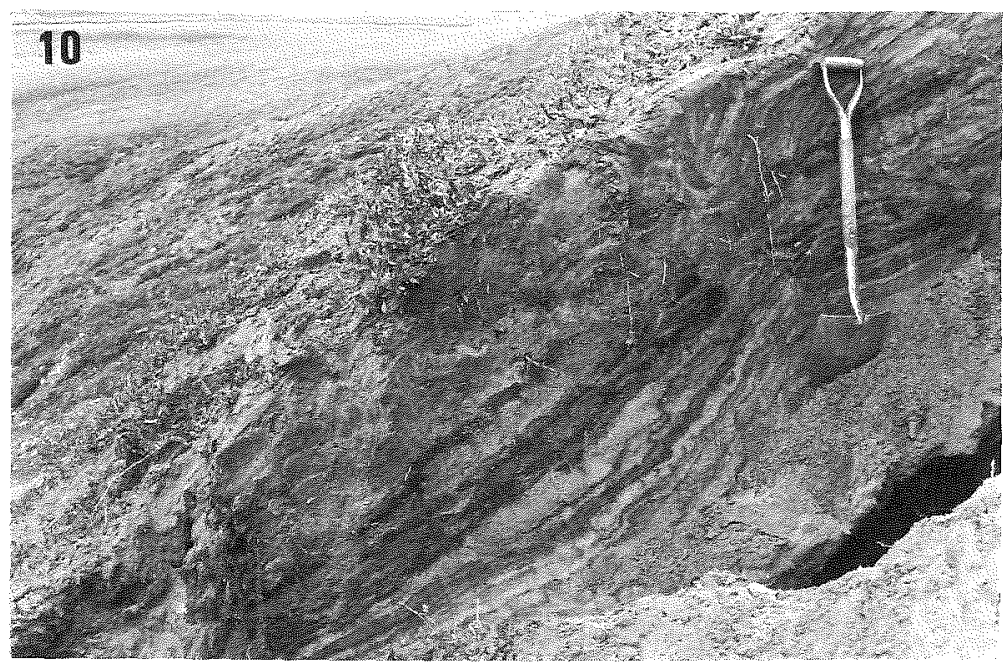

Frg. 10. Couches de sables éoliens mise en place sur un versant raide incliné de $40^{\circ}$, en bordure de la rivière Able. Localisation: site 9 de la fig. $3 \mathrm{D}$. La photo est prise en direction du nord. L'est est à gauche de cette photo.

sol par formation de glace de ségrégation, développement de fentes de gel, puis de cuvettes thermokarstiques, enfin perturbation de cet ensemble par des phénomènes de ravinement apparus en suivant le réseau de fentes de gel.

Les dépôts éoliens dont nous avons décrit les processus évolutifs étaient tous localisés sur de basses terrasses, très près de la Thomsen. A la localité 9 de la fig. 3D, en creusant une excavation sur un versant très raide, incliné actuellement de $40^{\circ}$, nous avons retrouvé des sédiments éoliens dont l'origine est aisément reconnaissable par le litage irrégulier d'horizons humifères, dû à la couverture végétale qui fixait le sable amené par le vent. Ce dépôt s'est mis en place sur un versant incliné de $36^{\circ}$ comme en témoigne la pente de la stratification (fig. 10).

\section{Etude granulométrique}

Une quinzaine d'échantillons provenant des sites $2,3,4,6,7,8$ et 10 de la fig. 3D ont été étudiés. Ces échantillons provenaient de sables éoliens prélevés des coupes et des surfaces actuellement actives (échantillons d à o) ainsi que de la formation d'Isachsen (échantillons a et b) et d'alluvions de la rivière Thomsen (échantillon c). Les courbes granulométriques de ces échantillons sont présentées dans la fig. 11 et quelques indices granulométriques qui les caractérisent sont rassemblés dans le tableau 3.

Les sables d'Isachsen (échantillons a et b) sont bien classés. Les deux échantillons examinés montrent qu'il existe cependant en leur sein des variations granulométriques appréciables. Les deux échantillons étudiés, qui ont été prélevés au hasard, ne permettent pas d'avoir une vue d'ensemble de la granulométrie de cette formation. Ils montrent toutefois que les sédiments de la formation d'Isachsen peuvent être considérés comme sources principales des sables éoliens de cette région.

Les courbes granulométriques des sables éoliens témoignent généralement d'un excellent classement. En prélevant les éléments les plus grossiers, c'est-à-dire ceux restant sur place après que la déflation ait remis en mouvement les sables qui les entouraient, nous avons obtenu la courbe $g$ (fig. 11). Au même endroit 7 de la fig. $3 \mathrm{D}$, un prélèvement des couches les plus fines, cohérentes, a donné la courbe h. Ces deux courbes nous paraissent représenter les extrêmes granulométriques des dépôts éoliens de la région.

Ces deux échantillons prélevés à la même place montrent bien l'existence de variations granulométriques importantes au sein du même sédiment. Ils indiquent que peuvent exister interstratifiés ensemble des fractions transportées par saltation et par suspension. Par ailleurs, les échantillons ont généralement été prélevés très près du cours de la rivière; seuls les prélèvements $\mathrm{n}$ et o s'en écartent de plusieurs centaines de mètres. Le transport éolien s'est donc exercé le plus souvent sur une faible distance. L'arrêt des éléments en saltation a généralement été provo- 


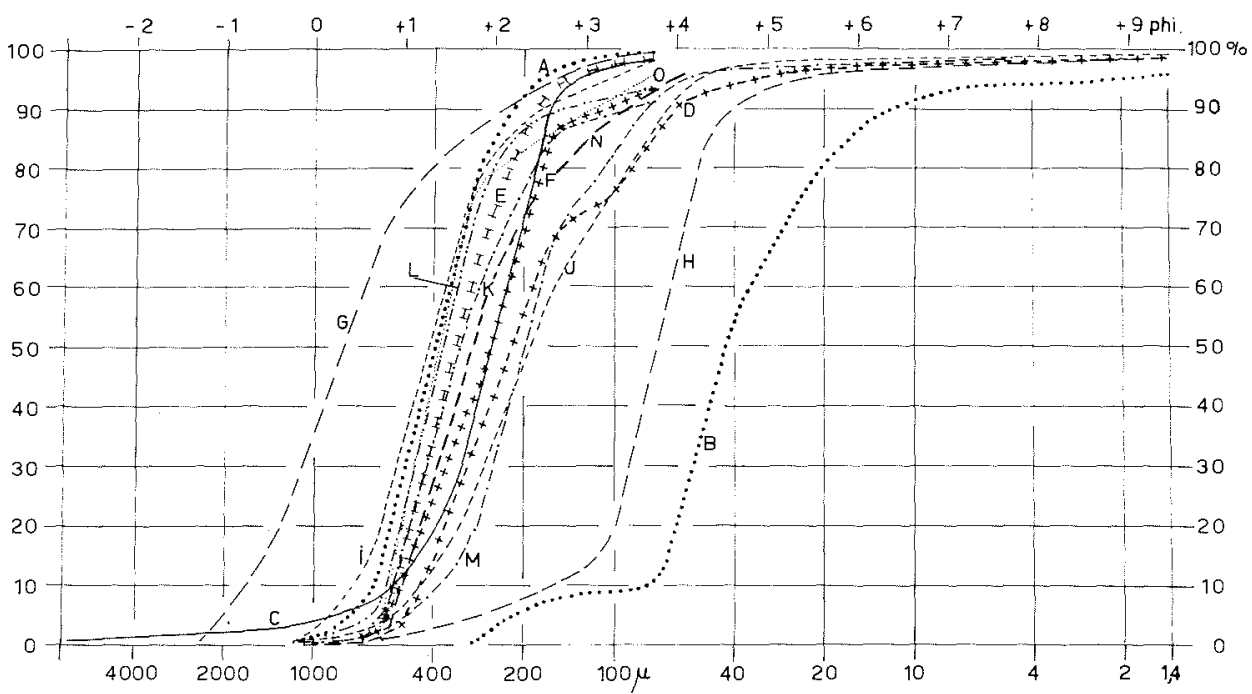

FIG. 11. Courbes granulométriques des sédiments prélevés près de la rivière Thomsen. Les lettres inscrites à côté de chaque courbe renvoient au tableau 3. La localisation des prélèvements est donnée sur la fig. 3D.

qué par la couverture végétale et s'est donc réalisé sans que s'effectue un tri granulométrique très élevé.

Malgré ces conditions défavorables, les courbes granulométriques sont en général caractéristiques des dépôts éoliens selon les critères proposés par Moiola et al. (1968) pour distinguer les sables fluviatiles et éolisés (fig. 12).

\section{Analyses polliniques}

Un examen des pollens contenus dans plusieurs échantillons provenant de la coupe décrite dans la fig. 4 a été effectué par M. R.J. Mott du Laboratoire de paléoécologie de la Commission géologique du Canada. Sauf pour les Laîches (Cyperaceae), les échantillons contenaient peu de pollens d'âge quaternaire. Parmi ces derniers, il y avait des espèces exotiques comme Picea, Pinus, Betula et Alnus, ainsi que certaines espèces endémiques comme Salix et Dryas, des graminées et des herbes. Malheureusement la concentration des pollens était trop faible pour permettre une analyse pollinique détaillée. La présence dans les échantillons d'une quantité importante de pollens d'âge tertiaire et mésozoïque supporte l'idée déjà exprimée que les sables éoliens proviennent $\mathrm{du}$ remaniement de formations géologiques anciennes.

\section{Datations au radiocarbone}

Afin d'établir le moment à partir duquel l'action éolienne a débuté en divers endroits, quatre échantillons de matières organiques provenant de la base de séquences de sédiments éoliens ont été datés.

Une branche de saule, identifié par L. D. Gill de la Commission géologique du Canada, a été recueillie par le second auteur, à $6 \mathrm{~m}$ sous la surface, dans une coupe naturelle au contact des dépôts éoliens et des sables et graviers alluviaux de la terrasse inférieure de la rivière Thomsen. Le site daté se situe sur la rive ouest de la Thomsen à l'aval de l'embouchure de la rivière Muskox (fig. 3D). L'âge radiométrique est de $3790 \pm 90$ ans BP (GSC-2119).

Un âge radiocarbone de $3460 \pm 80$ ans $B P$ (GSC-2124) a été compté à partir de fragments de saules identifiés par M. Kuc autrefois de la Commission géologique du Canada et prélevés par H. M. French et le premier auteur, à la base d'une séquence de $8 \mathrm{~m}$ de sédiments éoliens. Ces sédiments sont situés sur la rive gauche de la rivière Thomsen $2400 \mathrm{~m}$ en aval de l'embouchure de la rivière Able (fig. 3D).

Des brindilles prélevées par le second auteur dans de la tourbe sur la rive est de la rivière Bernard (fig. 3C) sous une épaisseur d'au moins $2 \mathrm{~m}$ de sables éoliens et au contact des dépôts graveleux d'une basse terrasse ont donné un âge ${ }^{14} \mathrm{C}$ de $5800 \pm 180$ ans BP (GSC-2242).

Ces trois datations au radiocarbone donnent un âge minimal pour l'abandon des plaines inondables qui forment aujourd'hui les basses terrasses des rivières Thomsen et Bernard et 


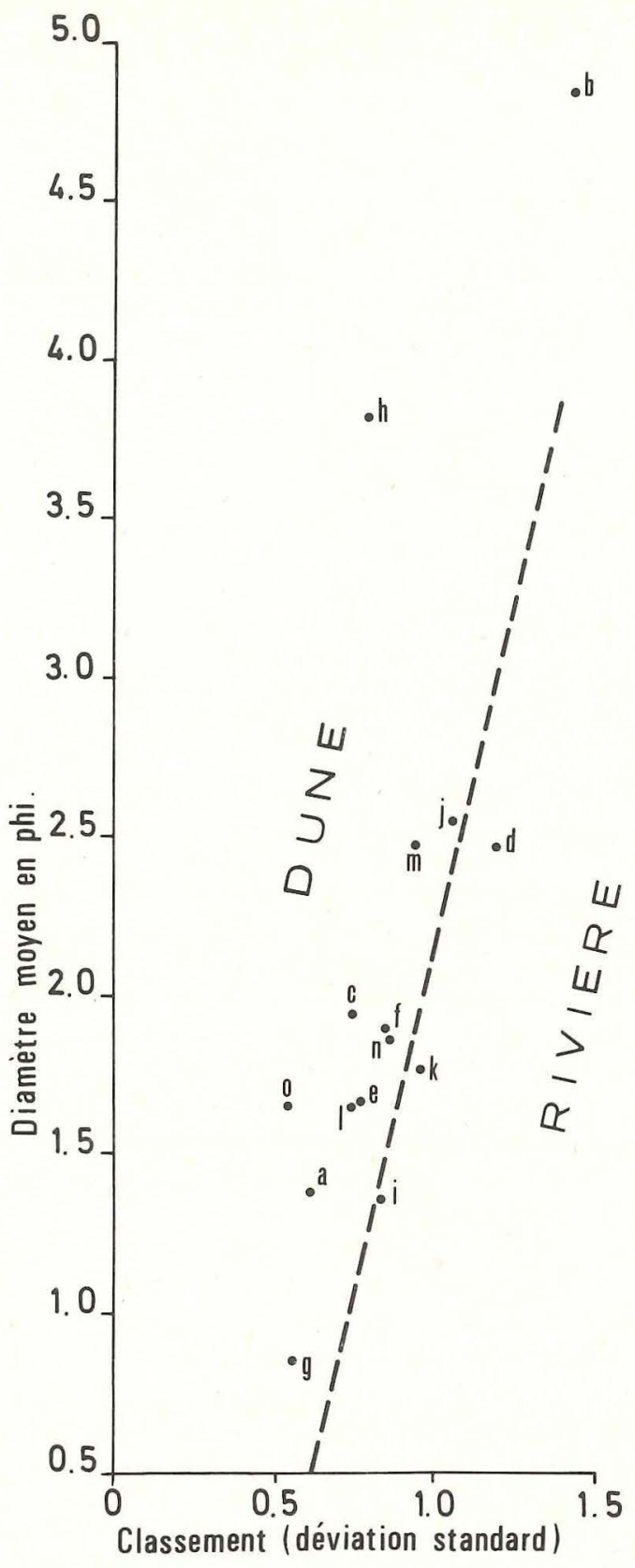

FIG. 12. Séparation selon Moiola et al. (1968) des sables dunaires et fluviatiles sur la base de leur granulométrie. Ces auteurs proposent la droite en tiretés. Les points inscrits correspondent aux échantillons de sable dont les caractères granulométriques sont donnés sur la fig. 11 et le tableau 3. La localisation des prélèvements est donnée sur la fig. 3D.

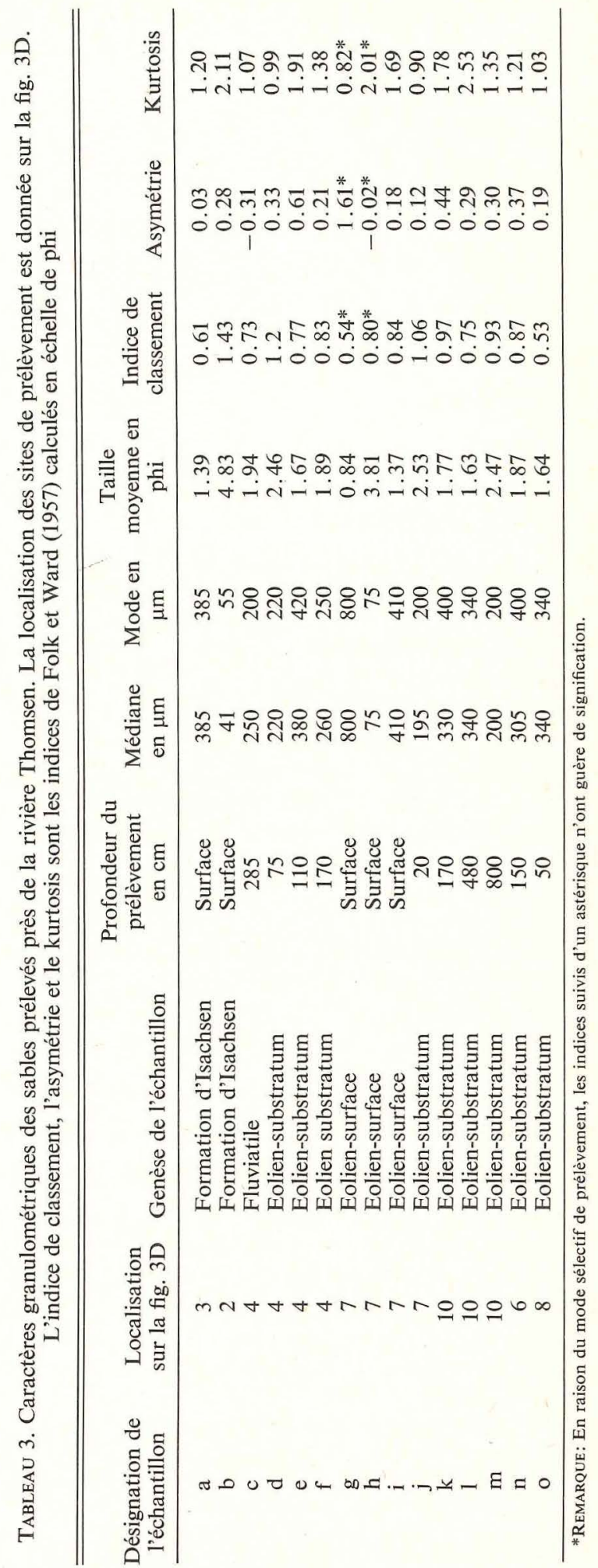


aussi pour le début de l'accumulation éolienne sur ces terrasses.

Des analyses polliniques de Ritchie et Hare (1971) appuyées par des datations au radiocarbone, suggèrent qu'une détérioration du climat a débuté il y a 5500 ans et est devenue très nette il y a environ 4000 ans dans la péninsule de Tuktoyaktuk à l'est du delta du fleuve Mackenzie. De même Nichols (1972) parle d'un refroidissement général dans l'Arctique canadien il ya a environ 3500 ans. Cette détérioration du climat aurait dû également affecter l'île de Banks, rendant l'environnement plus froid et plus aride.

Ces données indiquent qu'il existe une certaine correspondance entre les âges obtenus sur l'île de Banks pour le début de l'activité éolienne et le moment de la détérioration du climat dans l'Arctique signalée par Ritchie et Hare (1971) et par Nichols (1972). Il est également à noter que Rickert et Tedrow (1967) signalent que l'initiation de l'activité éolienne, à un endroit à proximité de la rivière Ikpikpuk sur la plaine côtière arctique en Alaska, a été datée à $3840 \pm 140$ ans BP (I-1004).

Bien que d'autres recherches soient nécessaires, il est intéressant d'avancer l'hypothèse que, et l'érosion verticale des plaines inondables, et le déclenchement de l'activité éolienne auraient pu être produites par des variations climatiques.

Selon Day de la Commission géologique du Canada (T. Day, communication personnelle 1976), l'avènement d'un climat plus froid et sec peut modifier le débit et la charge des cours d'eau et provoquer leur incision dans les plaines inondables. Bien que la masse totale d'eau précipitée à l'intérieur d'un bassin soit moindre, l'érosion verticale des cours d'eau peut être déclenchée ou être accélérée parce qu'une diminution de la charge de fond a pour conséquence une capacité érosive accrue des cours d'eau (McDonald et Lewis 1973, p. 41).

De même, une variation climatique peut être responsable du déclenchement de l'activité éolienne. Un climat plus aride permet à la surface du sol d'être asséchée pendant une plus longue période au cours de l'été et d'être ainsi apte plus longtemps à être mobilisée par le vent. Un climat plus froid modifie la végétation en provoquant la disparition de certaines espèces et en diminuant la densité du couvert végétal, améliorant ainsi les conditions de prise en charge des sédiments par le vent. Finalement la présence d'un climat plus aride cause une réduction des débits des cours d'eau, prolongeant ainsi la période d'étiage en été, ce qui a comme effet de faciliter la mobilisation éolienne des sédiments fins répartis sur les plaines alluviales.

Des variations climatiques ne sont cependant pas indispensables pour qu'apparaissent les phénomènes éoliens décrits plus haut. Des facteurs locaux tels que la largeur de la plaine alluviale, la hauteur et l'inclinaison des berges, l'orientation du lit par rapport aux vents violents peuvent déclencher ou arrêter les actions éoliennes qui se produisent dans les lits des rivières de l'île de Banks. D'autres datations sont nécessaires pour décider définitivement si une variation climatique est réellement intervenue.

Finalement, de la tourbe prélevée par le second auteur, à la base d'une séquence de $3.5 \mathrm{~m}$ de sédiments éoliens reposant sur les dépôts de la plaine d'épandage fluvio-glaciaire sise au sud-est de Sachs Harbour (fig. 3A), a donné un âge radiocarbone de $8430 \pm 120$ ans BP (GSC2419). Cette datation fournit un âge minimal pour le début de l'accumulation éolienne qui aurait succédee à la mise en place de la plaine d'épandage fluvio-glaciaire.

\section{Taux d'accumulation}

Les datations permettent d'évaluer le taux d'accumulation des sables éoliens. En admettant une activité régulière jusqu'à aujourd'hui, la séquence en aval de l'embouchure de la rivière Muskox correspond à un taux moyen d'accumulation de 1.55 à $1.62 \mathrm{~mm} /$ année, celle en aval de l'embouchure de la rivière Able à un taux de 2.26 à $2.37 \mathrm{~mm} /$ année, celle de la rivière Bernard à un taux moyen minimal de 0.33 à $0.36 \mathrm{~mm} /$ année et celle de la plaine d'épandage au sud-est de Sachs Harbour à un taux de 2.37 à 2.44 $\mathrm{mm} / \mathrm{année}$. Les résultats de 8 datations au radiocarbone permettent, selon Péwé (1975, p. 42), d'affirmer que le taux d'accumulation du loess au centre de J'Alaska varie entre 0.15 et $2.07 \mathrm{~mm}$ / année; Dyke (1976) a de son côté calculé, dans le cas de 14 sites différents, que les sables et silts éoliens sur l'île de Baffin s'accumulaient à un taux variant entre 0.13 et $3.00 \mathrm{~mm} /$ année. Les taux moyens d'accumulation sont respectivement pour les sites de l'île de Banks, de l'Alaska et de l'île de Baffin de $1.67,1.05$ et $0.77 \mathrm{~mm} /$ année. 


\section{Conclusions}

Nous avons montré que des dépôts éoliens non négligeables existaient sur l'île de Banks. Les phénomènes éoliens actuels y sont cependant étroitement localisés; ils n'ont pas l'importance qui leur a été parfois attribuée dans le haut Arctique canadien.

Le premier facteur quil limite l'action du vent est, même dans ces régions de haute latitude, la courverture végétale. En effet, sur l'île de Banks, comme dans les régions les plus septentrionales du Canada, la couverture végétale n'est presque jamais complètement absente. Les plantes, bien que souvent éparses, fixent efficacement les sables et ne permettent pas à la déflation de s'exercer. La grande quantité de débris organiques observés au sein des dépôts éoliens que nous avons décrits et leur disposition en lits continus horizontaux montrent clairement que la végétation a grandi au fur et à mesure de l'accumulation des dépôts et a contribué à leur fixation. Les travaux de Bagnold (1965, p. 183) ont établi que dans de telles conditions des dunes ne pouvaient pas se former; c'est sans doute la raison pour laquelle aucun relief dunaire n'a été trouvé sur l'île de Banks.

Ajoutons cependant que pendant l'hiver du sable est transporté sur la couverture de neige. Il peut ainsi être déplacé au-dessus d'un sol couvert de végétation. Ce processus permet au sable de s'étaler plus largement l'hiver que l'été. A la fin de l'hiver toutefois, lorsque la neige a fondu, ce sable arrive cependant sur la couverture végétale qui le fixe.

La végétation n'est pas le seul facteur qui arrête la déflation. Lorsque les sables contiennent aussi des cailloux, comme c'est par exemple le cas pour les sables et graviers de la formation de Beaufort, la concentration en surface d'éléments grossiers, cailloux et gravillons, arrête l'érosion éolienne. Dans des sédiments de granulométrie plus fine, c'est souvent la cohésion des particules fines qui s'oppose à l'érosion éolienne.

Les conditions sont très différentes dans les lits des rivières à chenaux anastomosés où bien entendu n'existe pas de végétation et où la crue de printemps abandonne chaque année à la surface des sables et des silts dépourvus de toute fraction argileuse. Celle-ci a en effet été emportée par les eaux des rivières. Les larges lits fluviatiles sont des sites extrêmement favorables à la déflation. Ainsi s'explique pourquoi dans les îles de
1'Arctique canadien, les actions éoliennes ont jusqu'à présent été effectivement observées à proximité des rivières: St-Onge (1965) a vue une tempête de poussières au cours de l'été 1959 dans la région deltaïque à l'est d'Isachsen sur l'île Ellef Ringnes et Pissart (1966) a signalé la présence de dépôts éoliens à proximité des plaines alluviales sur l'île Prince Patrick. Sur l'île de Banks, ce double remaniement des sédiments, tout d'abord par les rivières puis par le vent, a une importance considérable et cette observation est en complet accord avec les observations réalisées dans d'autres régions périglaciaires (par exemple Hobbs 1931; Péwé 1955) et dans les régions où a régné un climat périglaciaire en Europe occidentale.

Un dernier facteur mériterait d'être étudié avec attention, c'est la puissance des vents. Pissart avait montré, en 1966, que sur l'île Prince Patrick les vents étaient en général moins violents qu'on ne l'avait supposé. Les conditions sur Banks sont différentes, mais les observations météorologiques sont toujours insuffisantes pour apprécier l'importance de ce facteur.

\section{Remerciements}

Cette étude a été réalisée dans le cadre des projets 640004 et 740065 de la Commission géologique du Canada. Une bourse du Conseil des Arts du Canada a également permis au premier auteur de travailler dans l'Arctique pendant l'été 1974. Sur le terrain nous avons reçu un support matériel complet de la Commission géologique du Canada et de l'Etude du plateau continental polaire. Nous tenons à remercier les responsables de ces organismes pour l'aide accordée. Marc St-Onge, en qualité d'assistant, a généreusement aidé le premier auteur pendant tout son séjour dans l'Arctique. Nous assurons également de notre reconnaissance deux membres de la Commission géologique du Canada, à savoir M. W. Blake, Jr. pour les datations au radiocarbone, et $\mathrm{M}$. R.J. Mott pour l'étude palynologique d'échantillons qu'il a prélevés en notre compagnie dans une séquence de sédiments éoliens proche de la rivière Thomsen. MM. P. David de l'Université de Montréal et M. D. St-Onge de l'Université d'Ottawa ont aimablement lu et corrigé le manuscrit.

Bagnold, R. A. 1965. The physics of blown sand and desert dunes. Methuen and Company Limited, London, England, $265 \mathrm{p}$ 
BRD, B. J. 1967. The physiography of Arctic Canada with special reference to the area south of Parry Channel. The Johns Hopkins Press, Baltimore, MD, $336 \mathrm{p}$.

Corbel, J. 1958. La neige dans les régions hautement polaires (Canada, Groënland) au-delà du $80^{\circ}$ de latitude N. Revue de Géographie Alpine, \$6, pp. 343-366.

DRURY, W. H. JR. 1962. Patterned ground and vegetation on southern Bylot Island, Northwest Territories, Canada. Gray Herbarium Contribution 190, Harvard University, Cambridge, MA, $111 \mathrm{p}$.

DYKE, A. S. 1976. Quaternary geomorphology, glacial chronology, climatic history and sea level history of southwestern Cumberland Peninsula, Baffin Island, Northwest Territories, Canada. Thèse doctorale, University of Colorado, Boulder, $\mathrm{CO}$.

Embleton, C. et King, C. A. M. 1968. Glacial and periglacial geomorphology. Edward Arnold, London, England, $608 \mathrm{p}$.

FolK, R. L. et WARD, W. C. 1957. Brazos river bar - A study of the significance of grain size parameters. Journal of Sedimentary Petrology, 27, pp. 3-27.

FRENCH, H. M. 1972. The role of wind in periglacial environments, with special reference to northwest Banks Island, Western Canadian Aretic. Dans International Geography 1972 - La Géographie internationale. Editée par W. P. Adams et F. M. Helleiner. Communications présentées au 22e Congrès international de géographie, Canada, pp. 82-84.

1976. The periglacial environment, Longman Group Limited, London, England, 309 p .

French, H. M. et EgGinton, P. 1973. Thermokarst development, Banks Island, Western Canadian Arctic. North American Contributions, Permafrost Second International Conference. National Academy of Sciences, Washington, DC, pp. 203-212.

HовBS, W. H. 1931. Loess, pebble bands and boulders from glacial outwash of the Greenland continental glacier. Journal of Geology, 39, pp. 381-385.

KatAsonov, E. M. 1967. Features of deposits formed under permafrost conditions. Dans Arctic and alpine environments. Editée par H. E. Wright, Jr. et W. H. Osburn. Indiana University Press, p. 237-240.

Kuc, M. 1974. Noteworthy vascular plants collected in southwestern Banks Island, N.W.T. Arctic, 28, pp. $146-150$

MACKAY, J. R. 1972. The world of underground ice. Annals of the Association of American Geographers, 62, pp. I-22.

Mason, W. R. M., Shewell, G. E. et Cody, W. J. 1972. A plant collection from the southern interior of Banks Island, N.W.T. Canadian Field Naturalist, 86, pp. $363-367$.
McDonald, B. C. et Lewis, C. P. 1973. Geomorphic and sedimentologic processes of rivers and coasts, Yukon coastal plain. Environmental-Social Committee Northern Pipelines, Task Force on Northern Oil Development, Rapport No. 73-39, 245 p.

Molola, R. J., Phillips, B. J. et Weiser, D. 1968. Differentiation of beach, river and inland dune sands by whole-phi textural parameters. Oklahoma Geological Notes, 28, pp. 61-64.

Nichols, H. 1972. Summary of the palynological evidence for late-Quaternary vegetational and climatic change in the Central and Eastern Canadian Arctic. Dans Climatic changes in Arctic areas during the last ten-thousand years. Editée par Y. Vasari, H. Hy värinen et $S$. Hicks. Proceedings of a symposium held at Oulanka and Kevo, Finland, Oct. 1971. Acta Universitatis Ouluensis, Series A, Scientiae Rerum Naturalium no. 3, Geologica no. 1, pp. 309-339.

PÉWÉ, T. L. 1955. Origin of the upland silt near Fairbanks, Alaska. Bulletin of the Geological Society of America, 66, pp. 699-724.

1975. Quaternary geology of Alaska. United States Geological Survey, Professional Paper 835, $145 \mathrm{p}$.

PISSART, A. 1966. Le rôle géomorphologique du vent dans la région de Mould Bay, île Prince Patrick. Zeitschrift für Geomorphologie, 10, pp. 226-236.

1975. Glace de ségrégation, soulèvement du sol et phénomènes thermokarstiques dans les régions à pergélisol. Bulletin de la Société Géographique de Liège, 11, pp. 89-96.

Rickert, D. E. et Tedrow, J. C. F. 1967. Pedological investigations on some aeolian deposits of northern Alaska. Soil Science, 104, pp. 250-262.

Ritchie, J. C, et HARE, F. K. 1971. Late Quaternary vegetation and climate near the Arctic tree line of northwestern North America. Quaternary Research, 1 , pp. 331-342.

ST-ONGE, D. A. 1965. La géomorphologie de l'île Ellef Ringnes, Territoires du Nord-Ouest, Canada. Etude géographique, no 38, Direction de la géographie, Ministère des mines et des relevés techniques, Ottawa, $58 \mathrm{p}$.

Tricart, J. et Cailleux, A. 1961. Le modelé périglaciaire. Cours de géomorphologie. C.D.U., Paris $350 \mathrm{p}$.

VoN Thannheiser, D, et SCHWeIngRuber, F. 1974. Floristiche studien auf Banks Island, N.W.T. Polarforschung, 44, pp. 27-34.

WASHBURN, A. L. 1947. Reconnaissance geology of portions of Victoria Island and adjacent regions: Arctic Canada. Geological Society of America, Memoir 22, $142 \mathrm{p}$. 